\title{
Improvement of Therapy Outcomes after Negative Pressure Wound Therapy in a Patient with Acne inversa
}

\author{
Wojciech Francuzik $M D$, Tomasz Banasiewicz MD, Ph.D., Zygmunt Adamski MD, Ph.D.
}

\begin{abstract}
Treatment of acne inversa (also known as hidradenitis suppurativa) is complicated and chronic. This debilitating, inflammatory disease of the follicular sacks affects mostly young adults and has a strong negative impact on their quality of life. We present a case of a 28 year old woman with a history of acne inversa of Hurley grade 2 for 6 years. Patient underwent surgical excision of the skin of the left inguinum followed by negative pressure therapy dressings for 2 and a half weeks (5 dressing changes). This allowed a full closure of the wound after 12 weeks and formation of a well accepted scar. Patient's pain decreased from 4.5 to 1.5 according to visual assessment scale. We also noted a 28 point decrease in disease severity score according to Sartorius scale and a 19 point decrease in Dermatology Life Quality Index. Two years prior admission patient had undergone surgical treatment of her right inguinum with split thickness skin grafting, which healed for 26 weeks and yielded less satisfactory results. Comparison photographs of both treatment results are presented.
\end{abstract}

Keywords-Acne inversa, Hidradenitis suppurativa, Negative Pressure Wound Therapy

\section{INTRODUCTION}

Acne inversa (AI) is considered underdiagnosed and affects $1 \%$ of the population. ${ }^{1}$ Pathology of this debilitating inflammatory disease, also known as hidradenitis suppurativa is not well understood. Novel findings consider AI a disorder of follicular occlusion.2-4] Jansen et al. discussed nomenclature controversy of this disease and concluded that the term hidradenitis suppurativa is obsolete ${ }^{[5]}$ We recommend using the term acne inversa to avoid further confusion.

AI is most often localized in non-facial regions - the axillae, groin, anal folds, mons pubis, and scalp. It presents first as nodules and abscesses which can further penetrate deep into the skin forming fistulae and cause scarring. Pain and foul smell of the discharge make this disease unpleasant and significantly lower patient's quality of life. Continuous inflammation of the affected skin leads to fibrosis. Grading of the disease severity was classified by Hurley ${ }^{6}$ and further by Sartorius 7 and Revuz.

Treatment of AI with topical antiseptics and antibiotics may provide relief in early stages of the disease but relapses are frequent after withdrawal of medication therapy $\left[{ }^{5}\right.$ Therefore

Manuscript received 03.08.2014; revised 20.10.2014. No conflict of interest Author affiliation: Department of Dermatology, Heliodor Swiecicki Hospital in Poznań, Poland, (WF, ZA); Department of General Surgery, Heliodor Swiecicki Hospital in Poznań, Poland, (TB)

*Correspondence to: Wojciech Francuzik (e-mail: wojciech.francuzik@gmail.com). surgery is considered as the only reliable option in providing long term remission.

The purpose of this paper is to report a successful treatment of acne inversa in the inguinal region with wide excision of skin lesion coupled with subsequent use of negative pressure wound therapy (NPWT). To our best knowledge only 7 reports of negative pressure devices used in the treatment of AI exist in the literature. 12

We had a rare opportunity to compare therapy results of a previous surgical intervention that did not involve negative pressure therapy with those reinforced by vacuum assisted closure in this patient, what makes this paper of particular importance.

\section{CASE REPORT}

28 year old female, non-smoking patient was admitted to surgical Clinic in order to treat chronic acne inversa. The condition was first diagnosed in 2007 by a dermatologist and was since then treated with clindamycin and amoxicicllin/clavulanate systematically. Due to unsatisfactory results of pharmacological treatment, patient underwent split thickness skin grafting in her right inguinum 2 years before admission. She stayed in the hospital for three weeks and recovered fully after 4 months post procedure.

On the day of admission to our clinic (24 months post previous surgical intervention) some local skin irritation with patches of superficial ulceration was still present on the surface of the graft. Acute inflammatory lesions with labia majora and perianal skin involvement have persisted in the left inguinum for over a year (see Fig. 11). Patient was broken and depressed due to previously unsatisfactory therapy results and progression of the disease. She described her pain level as 4-5 according to visual assessment scale - (VAS). Patient was asked to fill out Dermatology Life Quality Index (DLQI) questionnaire $^{13}$ to assess the impact which AI has on her life quality before surgical intervention and 180 days post surgery.

\section{A. Surgical intervention}

We decided to perform a wide resection of the inflamed skin with underlying subcutaneous tissue. The surface of the excised skin with external openings of the abscesses was planned and in three areas the abscesses with subcutaneous fistulas were excised as three separate excisions. The inflamed soft tissue was removed en bloc, leaving only the healthy 


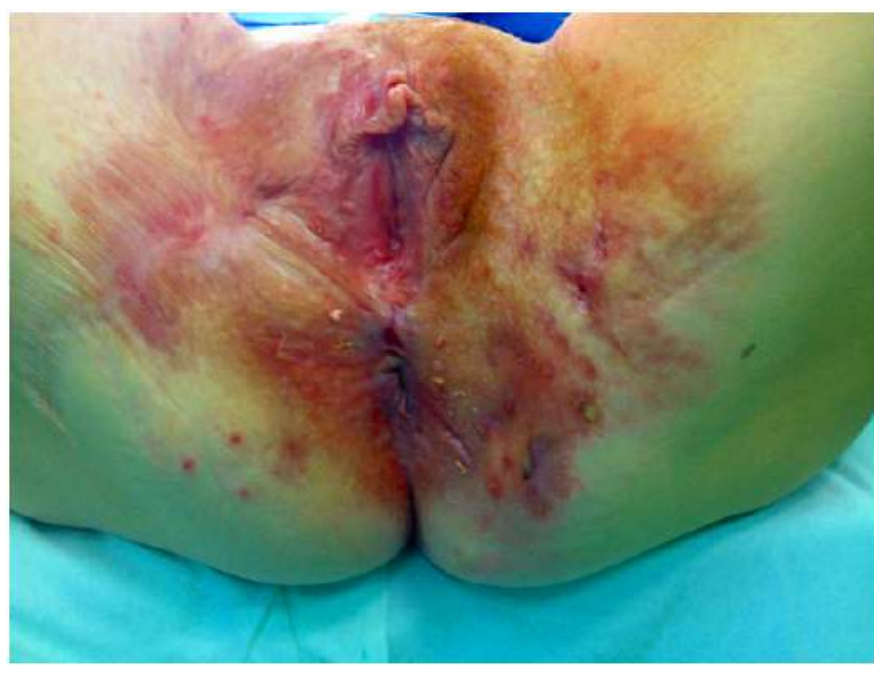

Fig. 1. Acne Inversa lesions in the left inguinum before treatment. Scars after previous wide excision with skin grafting visible in the right inguinum.

tissue. In the perianal region the excision was done up to the sphincter borders by careful electrocoagulation. The wound was washed after the procedure with $500 \mathrm{ml}$ of saline, followed by $200 \mathrm{ml}$ of octenidine. 30 seconds later the octenidine was washed out using another $500 \mathrm{ml}$ of saline. The subcutaneous sutures were located only in three points. A few cutaneous sutures were done, to avoid closing of the wound. In lowest point of the wound skin was leaved without sutures as an open wound. The drain located deep into the wound was inserted into a skin opening formed after excision of an abscess. The drain was closed and used temporary in next days to wash the wound with saline and octenidine 2 times daily. The polyurethane sponge was placed over the wound, with the silicon layer located directly on the surface of the wound as protection. Stoma paste was used to seal the dressing, especially in the anal area. In the next step the wound site with the polyurethane sponge was covered with external drape. The vacuum pad was located directly above the lower part of wound which remained open (see Fig. 2).

Negative pressure wound therapy (NPWT) was started (V.A.C. Therapy; KCI USA, Inc, San Antonio, Texas) using continuous method with negative pressure of $100 \mathrm{mmHg}$. In next days pressure was ranged from -85 to $-110 \mathrm{mmHg}$ depending on the volume of secretion. The wound was washed with saline and octenidine 2 times daily and wound dressing was changed every 2-3 days after previous administration of analgesics (ketoprofen $100 \mathrm{mg}$ orally). No complications or severe pain were observed. The patient described the therapy as fully acceptable and free of discomfort. Patient subjectively rated the course of treatment as more acceptable than during initial stay in 2012. The wound condition improved and after 3 dressing changes patient was sent home on the 8th day of hospitalization. Another 2 wound dressings were performed as an out-patient procedure. The first one was done after four days with the same device. Because of the lack of exudation we decided to switch to a portable device (PICO, Smith \& Nephew, London, UK). After another four days the vacuum therapy was discontinued. Patient applied the standard dressings and bathed
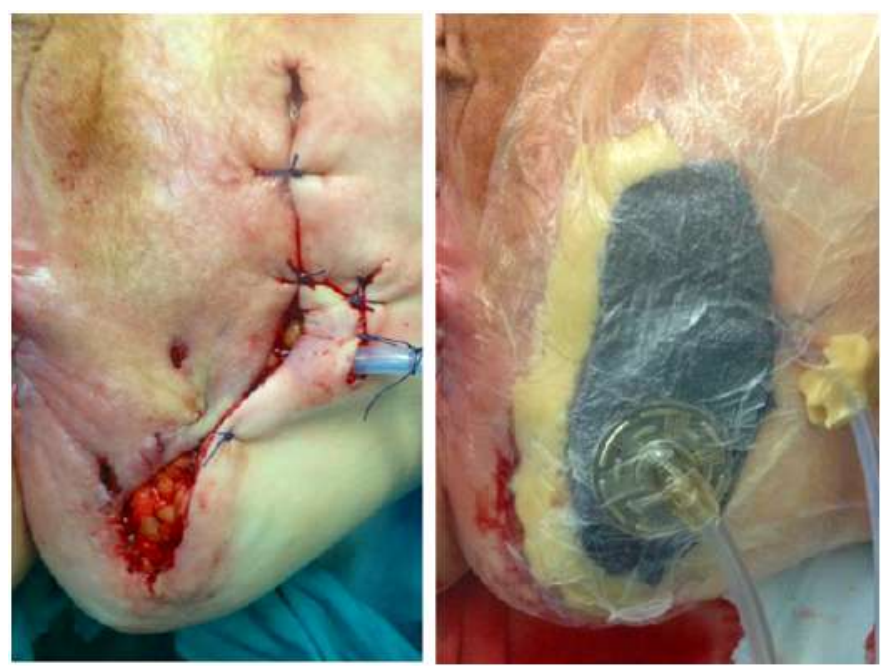

Fig. 2. Surgically treated left inguinum directly after excision of acne inversa lesions (left) followed by application of negative-pressure wound dressing (right).

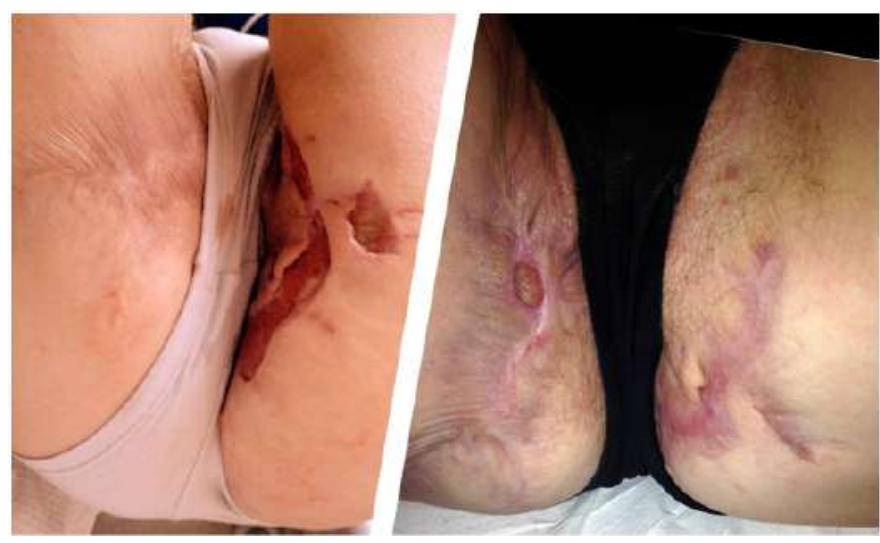

Fig. 3. Therapy outcome after 30 (left) and 90 (right) days post surgery.

the wound every day. The healing and granulation proceeded quickly, 4 weeks after surgery the patient returned to work, 6 weeks after surgery she stopped using dressings. During control visit after 90 and 180 days good results of the excision in the left inguinal region were observed, much better than in previously performed operation in the right inguinum (see Fig. 3). Assessment of clinical severity of the disease according to Sartorius scale ${ }^{7}$ revealed decrease of 28 paints compared to the state prior surgery (see table I). She reported only occasional mild pain (1,5 VAS). Her DLQI levels went down from 24 points (interpreted as extremely high impact on life quality) to 4 points (interpreted as small impact on life quality).

\section{DISCUSSION}

NPWT is a very effective and increasingly more common method of treatment of complicated wounds. It is applied in complicated fistulas, 14 and becomes an important part of complex plastic surgery procedures 15 The usefulness of NPWT in perineal and perianal localization is still limited, due to the technical difficulty of maintaining tightness and vacuum. Despite this, NPWT was successfully used in this localization 
TABLE I

ACNE INVERSA SEVERITY ACCORDING TO SARTORIUS SCALE IN A PATIENT TREATED SURGICALLY WITH SUBSEQUENT NEGATIVE PRESSURE DRESSINGS.

\begin{tabular}{lccccc}
\hline Region & Axilla & Groin & Gluteal & Inframmary & Other \\
\hline On admission & 11 & 39 & 4 & 0 & 5 \\
90 days post OP & 11 & 11 & 4 & 0 & 5 \\
\hline
\end{tabular}

for the treatment of the pilonidal cyst $\frac{16}{16}$ and complicated perianal injury ${ }^{17}$ Surgical treatment of AI is difficult because of the extension of the inflammation and its localization, which makes the resection uncomfortable for the patient.

The debate of best treatment modalities, is ongoing. The flap and split thickness skin grafts seem to be very common in surgical treatment. The literature provides scarce information on the use of NPWT in the treatment of AI. In patients treated with NPWT, vacuum is mostly used to improve the results of flap and skin graft healing. In our opinion, very good results are obtained when allowing to heal by second intention, combined with NPWT. This makes the procedures relatively easy to perform and time efficient, minimizes scarring and speeds up the process. It also minimizes the risk of potential complications inseparably connected with flap and skin grafts. Negative pressure facilitates faster granulation process and provides visually more acceptable outcomes as well as minimizes potential wound infections. It also improves the condition of surrounding tissue,and minimises potential skin contractures.

In order to assess therapy outcomes we used the Sartorius scale $e^{7}$ which is in our opinion the most comprehensive tool aviable for reporting treatment results in patients with AI.

Considering the overall good patient therapy response we were satisfied with the treatment outcomes. This method is fast and seems safer than allowing to heal by second intention, with an open wound susceptible to pathogen infiltration. This treatment seemed to manage skin lesions better than previously performed skin grafting in the right inguinal region.

Limitation of this study was that both procedures were performed with a significant time difference, making it harder to compare treatment results objectively. Also the patient did not consent to letting us perform control photographs after 150 days of treatment. We assure that the clinical state was very similar to the pictures taken 90 days post surgery.

Further research should focus on comparing therapy outcomes in acne inversa patients treated with the use of NPWT to those treated without such modality.

\section{Conclusion}

Our experience of surgical treatment of AI with wide excision followed by NPWT provides more benefits than solely allowing wounds to heal by second intention. Patient satisfaction is higher. Wound site is less likely to be infected and treatment results are faster achieved, what provides patients with a quicker return to normal life activities.

\section{REFERENCES}

[1] G. B. Jemec, M. Heidenheim, and N. H. Nielsen, "The prevalence of hidradenitis suppurativa and its potential precursor lesions," Journal of the American Academy of Dermatology, vol. 35, no. 2, pp. 191-194, 1996. [Online]. Available: http://dx.doi.org/10.1016/S0190-9622(96) 90321-7

[2] C. Yu and M. Cook, "Hidradenitis suppurativa: a disease of follicular epithelium, rather than apocrine glands," British Journal of Dermatology, vol. 122, no. 6, pp. 763-769, 1990. [Online]. Available: http://dx.doi.org/10.1111/j.1365-2133.1990.tb06264.x

[3] R. Attanoos, M. Appleton, and A. Douglas-Jones, "The pathogenesis of hidradenitis suppurativa: a closer look at apocrine and apoeccrine glands," British Journal of Dermatology, vol. 133, no. 2, pp. 254-258, 1995. [Online]. Available: http://dx.doi.org/10.1111/j.1365-2133.1995. tb02624.x

[4] J. Boer and E. Weltevreden, "Hidradenitis suppurativa or acne inversa. a clinicopathological study of early lesions," British Journal of Dermatology, vol. 135, no. 5, pp. 721-725, 1996. [Online]. Available: http://dx.doi.org/10.1111/j.1365-2133.1996.tb03880.x

[5] T. Jansen, P. Altmeyer, and G. Plewig, "Acne inversa (alias hidradenitis suppurativa)," Journal of the European Academy of Dermatology and Venereology, vol. 15 , no. 6, pp. 532-540, 2001. [Online]. Available: http://dx.doi.org/10.1046/j.1468-3083.2001.00303.x

[6] H. Hurley, "Axillary hyperhidrosis, apocrine bromhidrosis, hidradenitis suppurativa, and familial benign pemphigus: surgical approach," Dermatologic surgery. New York: Marcel Dekker, pp. 729-39, 1989.

[7] K. Sartorius, J. Lapins, L. Emtestam, and G. Jemec, "Suggestions for uniform outcome variables when reporting treatment effects in hidradenitis suppurativa," British journal of dermatology, vol. 149 no. 1, pp. 211-213, 2003. [Online]. Available: http://dx.doi.org/10. 1046/j.1365-2133.2003.05390.x

[8] J. Revuz, "Hidradenitis suppurativa," Journal of the European Academy of Dermatology and Venereology, vol. 23, no. 9, pp. 985-998, 2009. [Online]. Available: http://dx.doi.org/10.1111/j.1468-3083.2009.03356.x

[9] S. Ather, D. S. Chan, D. J. Leaper, and K. G. Harding, "Surgical treatment of hidradenitis suppurativa: case series and review of the literature," International wound journal, vol. 3, no. 3, pp. 159-169, 2006. [Online]. Available: http://dx.doi.org/10.1111/j.1742-481X.2006. 00235.x

[10] E. Chen and H. I. Friedman, "Management of regional hidradenitis suppurativa with vacuum-assisted closure and split thickness skin grafts," Annals of plastic surgery, vol. 67, no. 4, pp. 397-401, 2011. [Online]. Available: http://dx.doi.org/10.1097/SAP.0b013e3181f77bd6

[11] E. T. Elwood and D. G. Bolitho, "Negative-pressure dressings in the treatment of hidradenitis suppurativa," Annals of plastic surgery, vol. 46, no. 1, pp. 49-51, 2001. [Online]. Available: http://dx.doi.org/10.1097/00000637-200101000-00010

[12] P. J. Hynes, M. Earley, and D. Lawlor, "Split-thickness skin grafts and negative-pressure dressings in the treatment of axillary hidradenitis suppurativa," British Journal of Plastic Surgery, vol. 55, no. 6, pp. 507-509, Sep 2002. [Online]. Available: http://dx.doi.org/10.1054/bjps. 2002.3899

[13] A. Finlay and G. Khan, "Dermatology life quality index (DLQI)a simple practical measure for routine clinical use," Clin Exp Dermatol, vol. 19, no. 3, pp. 210-216, may 1994. [Online]. Available: http://dx.doi.org/10.1111/j.1365-2230.1994.tb01167.x

[14] T. Banasiewicz and M. Drews, "Negative pressure wound therapy (npwt) in open abdomen - an animal model for surgeons training," Negative Pressure Wound Therapy, vol. 1, no. 2, pp. 48-53, Apr 2014. [Online]. Available: http://researchpub.org/journal/npwt/number/ vol1-no2/vol1-no2-3.pdf

[15] S. Singh, "Role of negative pressure wound therapy in plastic surgery its basics, indications and contraindications," Negative Pressure Wound Therapy, vol. 1, no. 2, pp. 67-68, Apr 2014. [Online]. Available: http://researchpub.org/journal/npwt/number/vol1-no2/vol1-no2-6.pdf

[16] T. Banasiewicz, A. Bobkiewicz, M. Borejsza-Wysocki, M. Biczysko, A. Ratajczak, S. Malinger, and M. Drews, "Portable vac therapy improve the results of the treatment of the pilonidal sinus-randomized prospective study," Pol Przegl Chir, vol. 85, no. 7, pp. 371-3768, Jul 2013. [Online]. Available: http://www.degruyter.com/view/ j/pjs.2013.85.issue-7/pjs-2013-0056/pjs-2013-0056.xml;jsessionid= 57210FBBC4E59DFB6AE5D441E4D82C6F

[17] N. Horst, T. Banasiewicz, and P. Krokowicz, "Complex posttraumatic perineal wound with rectovaginal fistula - treatment with negative pressure therapy," Negative Pressure Wound Therapy, vol. 1, no. 1, pp. 17-21, Jan 2014. [Online]. Available: http://researchpub.org/journal/ npwt/number/vol1-no1/vol1-no1-4.pdf 